\title{
Otimizando o Processo Ensino e Aprendizagem com a Arquitetura para Desenvolvimento de Objetos de Aprendizagem - (ADOA)
}

\author{
Maici Duarte Leite ${ }^{1,2}$, Diego Marczal ${ }^{1,3}$, Eleandro Maschio ${ }^{1,3}$, \\ Andrey Ricardo Pimentel ${ }^{1}$, Alexandre Direne ${ }^{1}$, Francisco Reinaldo ${ }^{2}$ \\ ${ }^{1}$ Programa de Pós-Graduação em Informática \\ Universidade Federal do Paraná (UPFR) \\ Curitiba - PR - Brasil \\ ${ }^{2}$ Coordenação do Curso de Tecnologia em Sistemas para Internet \\ Universidade Tecnológica Federal do Paraná (UTFPR) \\ Guarapuava - PR - Brasil \\ ${ }^{3}$ Coordenação do Curso de Licenciatura em Informática \\ Universidade Tecnológica Federal do Paraná (UTFPR) \\ Francisco Beltrão - PR - Brasil \\ (maicileite, marczal, eleandrom, reinaldo) @utfpr.edu.br, (alexd, andrey)@inf.ufpr.br
}

\begin{abstract}
This article intends to propose an architecture for the creation of Learning Objects (LO) which employs the classification of mathematical errors with Multiple External Representations (MER), supported by ACT Theory. The architecture aims to supply more adequate error remediation for each student and also the interaction context. It is demonstrated by the development of a LO called Pythagoras. The experiment performed with real students certified the efficiency of the proposed architecture. The results indicated that the use of MER as a support for the errors remediations in LOs brings gains in knowledge acquisition by the learner.
\end{abstract}

Resumo. Este artigo propõe a Arquitetura para Desenvolvimento de Objetos de Aprendizagem (ADOA), empregando a classificação de erros matemáticos com Múltiplas Representações Externas (MRE), amparada pela Teoria Adaptive Control of Thought (ACT). A arquitetura objetiva prover remediações de erro mais adequadas a cada aluno ao contexto de interação. Ela é demonstrada por meio do desenvolvimento de um OA denominado Pitágoras. Experimentos realizados com alunos atestaram a eficiência da arquitetura proposta. Os resultados indicam que o uso de MRE como amparo à remediação de erros em OAs trazem ganhos significativos para aquisição de conhecimento do aprendiz.

\section{Introdução}

A utilização de materiais didáticos suportados pela Inteligência Artificial (IA) tem se tornado uma prática comum. A IA permite modelar as características do aluno, possibilitando a flexibilização do comportamento no ambiente durante as interações, 


\section{CBIE-LACLO 2015}

Anais do XXVI Simpósio Brasileiro de Informática na Educação (SBIE 2015)

ou seja, aplicam técnicas como a remediação a erros para orientar o aluno a corrigir seu erro após uma interação mal sucedida [Leite et al. 2013].

No que diz respeito aos Objetos de Aprendizagem (OAs), a remediação ${ }^{1}$ de erros tem sido aplicada de forma mais restrita. As tentativas de remediação, nesse caso, se concentram apenas em proporcionar feedback simples, por meio de mensagens, indicando se a interação está correta [Marczal and Direne 2011, Oliveira et al. 2012, Leite et al. 2013]. Diante disso, destaca-se a remediação de erros como uma das possibilidades presentes nos STIs que pode ser estendida aos OAs de modo a favorecer o processo de aquisição de conhecimento pelo aluno. Por sua vez, o termo Representação Externa (RE) foi criado para descrever o emprego de técnicas para apresentar, representar e organizar o conhecimento [Cox and Brna 1995]. Pesquisas sobre o uso de REs em sistemas educacionais confirmam que os alunos se beneficiam de RE, inclusive diminuindo a carga cognitiva envolvida [Rau and Scheines 2012].

O erro matemático e a classificação de erros tem sido explorado como possibilidade no processo de resolução de um aluno, que atrelado a conceitos presentes em STIs permite modelar, rastrear e corrigir as etapas do processo de resolução. Isso possibilita que seja feito um acompanhamento individualizado do aluno [Nunes and Jaques 2014]. Estudos [Marczal and Direne 2011, Bazzo et al. 2011, Oliveira et al. 2012, Leite et al. 2013, Leite et al. 2014] têm discutido esse assunto e apresentado algumas abordagens para que informações sobre o erro possam auxiliar o aluno no seu processo de aprendizagem. Dentre elas, há: (1) a possibilidade do aluno (ou professor) retroceder ao contexto exato de um erro; (2) a classificação de um erro em sub ou supergeneralização; (3) e também a remediação de erros por meio de Múltiplas Representações Externas ${ }^{2}$ (MREs).

O artigo aborda a aplicação de conceitos de STIs em OAs, composta por uma arquitetura que engloba: (1) a classificação de erros matemáticos; (2) a interseção com Múltiplas Representações Externas; (3) a remediação de erros; e (4) o uso de uma técnica baseada no model tracing, presente na Teoria Adaptative Control of Thought (ACT), de John Anderson [Anderson 1983]. Como validação, foi desenvolvido um OA, que explora o Teorema de Pitágoras por meio dela e, em contraste, outro OA com proposta tradicional (com perguntas e respostas, tendo feedbacks somente textuais). Os resultados indicam que a abordagem proposta traz benefícios ao aprendizado, tais como atingir menor tempo de resposta com maior número de questões resolvidas e em menor número de tentativas de respostas sem sucesso.

\section{Erros Matemáticos e as Múltiplas Representações Externas}

A classificação de erros matemáticos é crucial na apresentação de uma RE durante o processo de remediação de erros. Um estudo anterior categorizou os erros matemáticos [Leite et al. 2012]: (1) interpretação equivocada da linguagem: esse tipo de erro alerta para a dificuldade do aluno em avançar na compreensão da estrutura do problema antes mesmo de formular uma estratégia; (2) diretamente identificáveis:

\footnotetext{
${ }^{1}$ De acordo com o Dicionário Priberam da Língua Portuguesa: " 1. Dar remédio a. 2. Compor, corrigir, arranjar. 3. Impedir, evitar. 4. Socorrer, auxiliar. 5. Prover do que é indispensável". Para a pesquisa, remediar significa auxiliar o aluno a se recuperar de um erro.

${ }^{2}$ Do Inglês, Multiple External Representations
} 


\section{CBIE-LACLO 2015}

Anais do XXVI Simpósio Brasileiro de Informática na Educação (SBIE 2015)

abrange o erros vindos da deficiência no domínio, do uso inadequado de dados, da deficiência de regras, teoremas ou definições, ou da aplicação de operados lógicos; (3) indiretamente identificáveis: contempla o erro oriundo da falta de lógica correta, podendo ser uma classificação incorreta, uma resposta incorreta para uma estratégia, ou uma transformação que não resulte em avanço; (4) solução não-categorizável: inexistência de classificação entre as demais categorias citadas.

Optou-se por agregar os estudos de Ainsworth sobre MREs à classificação de erros apresentada, com objetivo de propor uma remediação de erros mais eficiente. O emprego de REs deve considerar as funções exercidas em situações de aprendizado [Ainsworth 2006], segundo a taxonomia: (1) função de papéis complementares, que explora a representação para apoiar ou complementar um processo cognitivo; (2) função restrição de compreensão, que restringe possíveis representações, que não sejam relevantes para determinados conceitos; e (3) função de construção de conhecimento aprofundado, que explora a possibilidade do uso de MREs para a criação de uma compreensão aprofundada, obtida pela generalização de regularidades.

\section{ACT - Adaptive Control of Thought}

O ACT [Anderson 1983] constitui-se de uma teoria unificada do processamento de informações, onde os mecanismos de aprendizagem estão estritamente relacionados a processos, especialmente com a forma como se apresenta a informação e admite como princípios: (1) identificar a estrutura do objetivo do espaço do problema, que pode acontecer com base em um questionamento; (2) fornecer instruções no âmbito da resolução de problemas, que pode ser na forma do acesso ao conceito formalizado do conteúdo a ser explorado; (3) fornecer feedback imediato sobre os erros, para que o aluno possa ser orientado ao longo do processo e não somente na etapa final; (4) minimizar a carga de trabalho de memória; (5) ajustar a granularidade da instrução com a aprendizagem para suportar o processo de compilação de conhecimento; e (6) capacitar o aluno para a abordagem da habilidade-alvo por aproximações sucessivas.

O modelo cognitivo, baseado na Teoria ACT, possui um sistema de regras de produção, que são capazes de gerar uma multiplicidade de passos para as soluções e equívocos de alunos. O modelo cognitivo é a base para duas técnicas de modelagem: model tracing e knowledge tracing.

O model tracing é um modelo cognitivo, que aborda o aluno individualmente em seu caminho na solução dos problemas que lhe são apresentados, interpretando cada ação e respondendo de forma específica. A possibilidade de alertar o aluno no momento em que se desvia da estratégia correta permite uma revisão da estratégia de resolução, passando pelo processo de pensamento ao invés de tentativa-e-erro.

O knowledge tracing visa determinar o conhecimento do aluno, tanto sobre seus acertos quanto sobre seus erros. Este modelo cognitivo tem emprego na avaliação do aluno, bem como na sugestão das suas próximos iterações, de modo a otimizar a aquisição de conhecimento.

Considerando os dois modelos, a arquitetura proposta pela pesquisa corrente se baseou no model tracing. Tal modelo se mostra mais aderente a alguns objetivos, como remediar erros por meio de MREs ou retroceder ao contexto exato de um erro. 


\section{Arquitetura Proposta para Construção de OAs com Remediação por MRE}

A correspondência da classificação de erros com as principais funções de MREs presentes na taxonomia de Ainsworth objetiva explorar cada RE no processo de remediação, de forma a potencializar a interação do aluno na sua busca pela solução do problema em tela. Assim, a proposta da remediação de erros apoiada por MRE intenciona manter o aluno no caminho correto de resolução, através da divisão de um problema em etapas. Isso é feito por meio da apresentação de uma $R E$ adequada a cada erro cometido pelo aluno no processo.

Com o amparo dessas REs em momento oportuno, espera-se que o aluno amplie a sua base de conhecimentos a partir da reflexão e da manipulação de informações sob novas perspectivas, não consideradas em um primeiro momento. Como exemplo, um aluno pode não ter sucesso em extrair dados de um enunciado para estruturar uma equação, entretanto poderia conseguir resolver a mesma equação, caso ela estivesse organizada em etapas de solução.

Além do suporte à classificação de erros correlacionada às funções de MREs, o processo que apresenta a remediação também buscou considerar aspectos como a persistência do erro condicionada ao número de tentativas e o sucesso ou fracasso com a RE anterior, condicionada à ação do aluno.

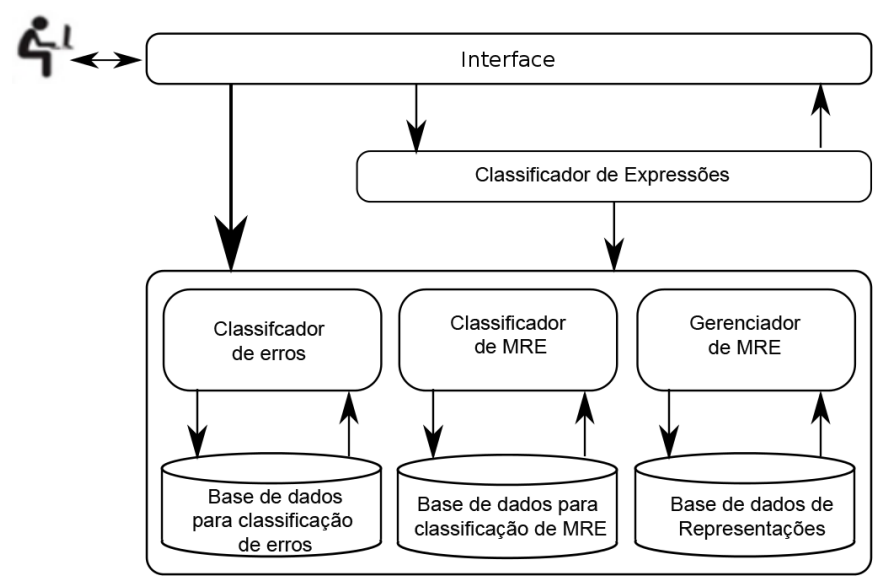

Figura 1. ADOA - Arquitetura para Remediação de erros com MREs em OAs

A Figura 1 apresenta a ADOA, que estrutura o processo de remediação de erros vinculado as funções de MREs, conforme abordagem mencionada.

A ADOA é composta por módulos a serem acionados caso o aluno erre. Assim, o módulo Classificador de Expressões recebe os dados para identificar acerto ou erro. Se um erro for detectado, o Módulo Classificador de Erro classifica o tipo de erro através das regras presentes no Módulo de Base de Regras da Classificação de Erro, que contém a classificação de erros. O módulo Classificador de Função de MRE tem como objetivo identificar o erro classificado e desencadear o processo de remediação de erros. O módulo Gerenciador de MRE definirá qual o tipo de remediação o aluno necessita para avançar em sua estratégia de resolução.

Na construção da ADOA, o processo de classificar um erro e de, então, 


\section{CBIE-LACLO 2015}

Anais do XXVI Simpósio Brasileiro de Informática na Educação (SBIE 2015)

relacioná-lo com REs [Leite et al. 2012], considerando suas funções, clarificou a estruturação do conjunto de REs que comporá o processo de remediação de erro.

\section{Construção do OA Pitágoras com a Arquitetura ADOA}

Para a validação da arquitetura proposta, foram construídos dois OAs, chamados de Pitágoras Mix e Pitágoras Max, respectivamente. Eles foram desenvolvidos com a ferramenta de autoria FARMA (Ferramenta de Autoria para Remediação de erros com Mobilidade na Aprendizagem) [Marczal and Direne 2011]. Trata-se de uma tecnologia que permite criar OAs para o ensino de Matemática com exercícios baseados no model tracing, da Teoria ACT, ou seja, que promovam a resolução em etapas. A FARMA também armazena todos os registros de erros e acertos dos alunos, de modo que alunos e professores possam voltar ao contexto exato da ocorrência de um erro, mesmo transcorrido um grande período de tempo após a tentativa de solução.

Para a realização do experimento, ambas as versões, Pitágoras Mix e Max, foram organizadas para apresentar os mesmos problemas matemáticos envolvendo o Teorema de Pitágoras. Entretanto, a versão Pitágoras Mix não contava com o modelo arquitetural proposto para a divisão dos exercícios em etapas, nem com o uso de MRE para a remediação de erros.

A Figura 2 apresenta o enunciado exibido ao aluno pelo objeto Pitágoras Mix. Em caso de equívoco do aluno, o OA somente retorna a mensagem "Incorreto".

Um ciclista acrobático deseja atravessar de um prédio ao outro com uma bicicleta especial, por meio de um cabo de aço rígido. O prédio de partida tem a altura de 75 metros e o de chegada tem a altura de 25 metros. A distância entre as duas torres é de 120 metros.

Qual é a medida mínima do comprimento do cabo de aço?

Figura 2. Enunciado do problema no OA Pitágoras Mix

Dessa forma, no Pitágoras Mix, o aluno teria de abstratir todas as etapas de resolução sozinho, sendo exigida maior capacidade de abstração. Tal fato pode ser destacado como uma dificuldade frequente dos alunos, ainda mais quando ocorre a apresentação de um novo conceito.

Em contrapartida, o mesmo exercício, apresentado pelo objeto Pitágoras Max, foi dividido em etapas, promovendo o acompanhamento do caminho de solução do aluno. Como antecipado, a Teoria ACT suporta o procedimento para organizar e acompanhar toda a interação do aluno com o Pitágoras Max. Ela atua no mecanismo de definição para que se apresente uma RE adequada àquele momento, auxiliando na remediação do erro em questão.

Assim, objetiva-se otimizar a trajetória do aluno para a aquisição de um conceito. Nesse sentido, identificado o caminho de solução de um determinado problema, o model tracing proporciona uma exploração individualizada da etapa em que o aluno se encontra do processo de resolução.

Portanto, o experimento com o Pitágoras Max contempla as características e propriedades da arquitetura proposta por esta pesquisa, explorando o processo 
de remediação de erros por meio de MRE, a partir de uma classificação de erros matemáticos. O enunciado da Figura 3 apresenta o exercício do Pitágoras Max dividido nas cinco etapas abaixo.

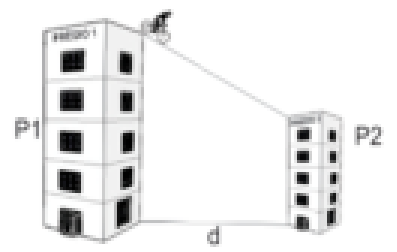

Figura 3. Enunciado do problema no OA Pitágoras Max

\begin{abstract}
Um ciclista acrobútico deseja atruvessar de um prédio a outro cam umo bicicleto especial par meio de um cabo de aço rigido. O prédio de partido tem $a$ altura de 75 metros e o de chegado tem a altura de 25 metros. A distáncia entre as duas torres é de 120 metros.
\end{abstract}

Etapa 1: Quais as medidas indicadas na figura? Resposta: 75; 25; 120.

Caso o aluno não responda corretamente à Etapa 1, aplicam-se as REs da Figura 4, objetivando a remediação dos erros apresentados, para três equívocos.

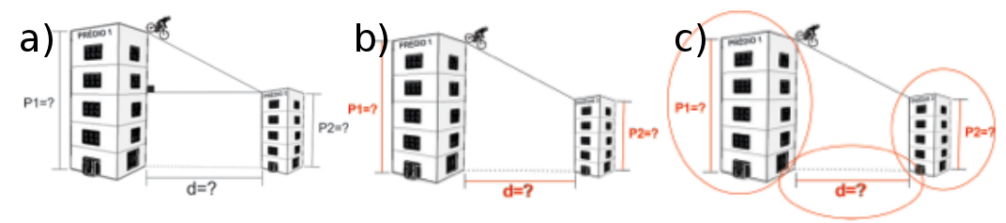

Figura 4. REs para remediação da Etapa 1

Etapa 2: Qual a diferença de altura entre as duas torres? Resposta: 50.

Na Etapa 2, espera-se que o aluno identifique os valores que comporão sua resposta final. Os possíveis equívocos são remediados com as REs da Figura 5.

a)

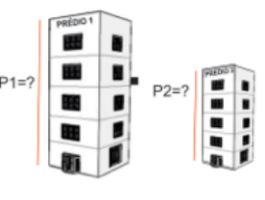

b)

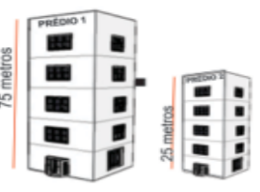

Figura 5. REs para remediação da Etapa 2

Etapa 3: Para descobrir o comprimento mínimo do cabo de aço que liga as duas torres é necessário extrair um triângulo retângulo da figura citada e então aplicar o Teorema de Pitágoras. Assim, quais seriam as medidas presentes no triângulo? Resposta: 50; 120.

Na Etapa 3, pretende-se explorar o Teorema de Pitágoras. Espera-se que o aluno tenha a compreensão do triângulo retângulo e suas abstrações. As REs da Figura 6 procuram remediar equívocos relacionados. 

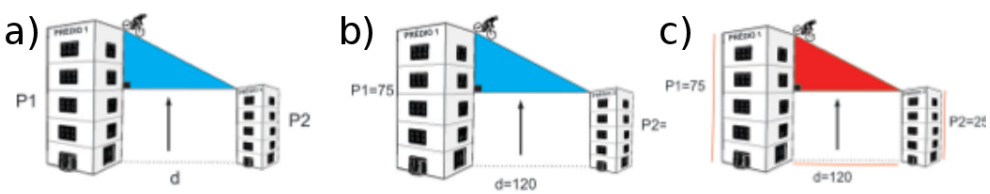

Figura 6. REs para remediação da Etapa 3

Etapa 4: Sabendo que o comprimento do cabo de aço é representado pela letra 'a', apresente a equação do Teorema de Pitágoras aplicada à figura do ciclista para descobrir o comprimento mínimo necessário do cabo de aço para ir de uma torre a outra. Resposta: $a^{2}=50^{2}+120^{2}$.

Na Etapa 4, espera-se que o aluno tenha abstraído a equação. Convém destacar que é comum outros equívocos no que diz respeito à posição dos termos dentro da equação. Eles são remediados pelas REs presentes na Figura 7.
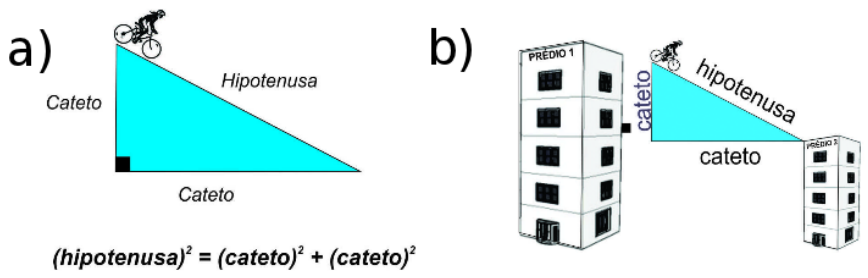

Figura 7. REs para remediação da Etapa 4

Etapa 5: Qual é o comprimento mínimo do cabo de aço necessário para ir de um prédio ao outro? Resposta: 130 .

Finalmente, na Etapa 5, o aluno pode concluir o exercícios, resolvendo a equação. Erros nesta última etapa são remediados pelas REs da Figura 8.

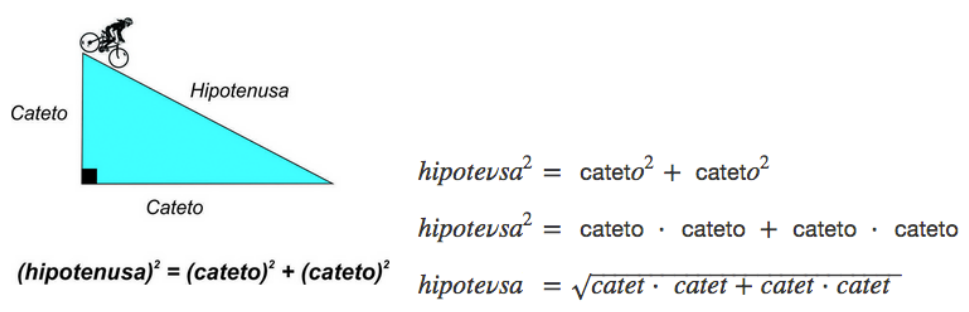

Figura 8. REs para remediação da Etapa 5

Isto posto, o Pitágoras Max oferece um aprendizado gradual por meio da estruturação de passos (transformados em etapas no OA), amparados pelos conceitos no model tracing da Teoria ACT. Cada erro apresentado é classificado, sendo também armazenada a etapa correspondente à ocorrência, havendo a validação da ordem do equívoco e posterior relação com as funções de MRE. Somente após todo este processo uma remediação de erro é sugerida. 


\section{CBIE-LACLO 2015}

Anais do XXVI Simpósio Brasileiro de Informática na Educação (SBIE 2015)

\section{Experimento}

A proposta é validar se a criação de um OA, utilizando a arquitetura proposta, oferece ganhos na aprendizagem, em relação a outro que não utilize a mesma arquitetura, mas que explore os mesmos conceitos. Para isso, os experimentos realizados foram compostos de três etapas: (1) pré-teste, que consistiu de uma avaliação escrita para que se definisse quais seriam os alunos presentes nos grupos de controle (GC) e experimental (GE); (2) utilização de ambos os OAs, Pitágoras Mix (pelo GC) e Max (pelo GE), pelos alunos; e (3) pós-teste, em termos de outra avaliação escrita. O experimento contou com 20 alunos com idade entre 15 e 22 anos.

O delineamento permitiu uma análise comparativa dos GC e GE por meio do teste $t$-student para amostras independentes. Os resultados indicam que o uso das MRE na remediação de erros pode trazer benefícios à aquisição de conhecimento. Detalhes dessa análise foram publicados em [Leite et al. 2013, Leite et al. 2014].

Ao presente artigo cabe analisar se, quando o aluno recebe uma remediação personalizada, de acordo com a correspondência do erro às MRE, seu processo evolutivo na resolução do problema tende a ser: (a) mais rápido; (b) mais completa, no sentido de resolver mais exercícios; e (c) com menos tentativas incorretas.

\section{Resultados}

Os resultados são apresentados por meio de um comparativo da completude, tempo gasto para responder corretamente uma questão e quantidade de tentativas por questão, dos OAs Pitágoras Mix (Turma A - TA) e Max (Turma B - TB).

Como observado na Tabela 1, os indivíduos que interagiram com o Pitágoras Max, considerados como Turma B (TB) resolveram mais questões do que aqueles que interagiram com o Pitágoras Mix, Turma A (TA). Em média, resolveram $76 \%$ (TB) das questões, contra 48,74\% do Pitágoras Mix (TA). Além disso, 11 alunos resolveram todas as questões (100\% de completude) no Pitágoras Max (TB).

Assim, os alunos que usaram o Pitágoras Max-TB resolveram as questões mais rápido (em média 3,1 min/questão) do que na versão Mix-TA (em média 9,4 min/questão). Os alunos que utilizaram o Pitágoras Max apresentaram um menor número de tentativas por questões, 1,933 contra 2,200. Esses dados destacam que as MREs, como recurso na remediação de erros, trazem, além de maior aquisição de conhecimento outras potencialidades a serem exploradas na resolução de problemas.

A possibilidade de dividir um problema em etapas, e de que em cada etapa seja possível apresentar uma remediação de erro apropriada, já parece ser um ganho significativo. Tal aspecto ganha mais relevância porque não se trata somente de uma remediação ao erro, mas de uma classificação do erro perante as funções de MRE. Em consequência, o aluno recebe um feedback bastante específico e personalizado.

O benefício continua se estendendo caso a remediação sugerida não acabe com o impasse do aluno, e incorra novamente no mesmo erro. Na ocorrência disso, uma outra RE é apresentada, com o propósito de amparar o processo de resolução, diminuindo a carga cognitiva.

As intervenções presentes no Pitágoras Max viabilizam o suporte antes da 
CBIE-LACLO 2015

Anais do XXVI Simpósio Brasileiro de Informática na Educação (SBIE 2015)

Tabela 1. Completude, tempo gasto para responder corretamente uma questão e quantidade de tentativas por questão do OA Pitágoras Mix

\begin{tabular}{|c|c|c|c|c|c|c|}
\hline \multirow{2}{*}{$\begin{array}{c}\text { Aluno } \\
\text { TA e TB }\end{array}$} & \multicolumn{2}{|c|}{ Completude (\%) } & \multicolumn{2}{|c|}{$\begin{array}{c}\text { Tempo médio de resposta } \\
\text { correta (min) }\end{array}$} & \multicolumn{2}{|c|}{ Média de respostas por questão } \\
\hline & TA & $\mathrm{TB}$ & TA & TB & TA & TB \\
\hline 01 & $75,00 \%$ & $100,00 \%$ & 8,333 & 2,000 & 1,250 & 2,417 \\
\hline 02 & $0,00 \%$ & $16,66 \%$ & 21,000 & 1,000 & 3,250 & 0,417 \\
\hline 03 & $75,00 \%$ & $58,33 \%$ & 2,667 & 4,000 & 0,750 & 1,250 \\
\hline 04 & $75,00 \%$ & $100,00 \%$ & 0,667 & 2,000 & 1,250 & 2,417 \\
\hline 05 & $25,00 \%$ & $100,00 \%$ & 3,333 & 2,000 & 1,500 & 1,750 \\
\hline 06 & $0,00 \%$ & $100,00 \%$ & 8,000 & 1,000 & 1,750 & 1,000 \\
\hline 07 & $50,00 \%$ & $100,00 \%$ & 19,000 & 1,000 & 3,750 & 1,500 \\
\hline 08 & $50,00 \%$ & $100,00 \%$ & 8,500 & 2,000 & 2,750 & 2,000 \\
\hline 09 & $50,00 \%$ & $100,00 \%$ & 8,000 & 2,000 & 2,000 & 1,417 \\
\hline 10 & $50,00 \%$ & $100,00 \%$ & 11,500 & 2,000 & 3,000 & 2,250 \\
\hline 11 & $75,00 \%$ & $50,00 \%$ & 1,667 & 3,000 & 0,750 & 2,000 \\
\hline 12 & $50,00 \%$ & $33,33 \%$ & 10,500 & 5,000 & 3,000 & 1,333 \\
\hline 13 & $75,00 \%$ & $41,66 \%$ & 3,667 & 6,000 & 1,250 & 3,083 \\
\hline 14 & $50,00 \%$ & $58,33 \%$ & 10,500 & 3,000 & 2,750 & 2,667 \\
\hline 15 & $25,00 \%$ & $25,00 \%$ & 21,000 & 1,000 & 3,500 & 0,250 \\
\hline 16 & $25,00 \%$ & $100,00 \%$ & 17,000 & 7,000 & 1,500 & 2,500 \\
\hline 17 & $75,00 \%$ & $58,33 \%$ & 3,333 & 5,000 & 1,750 & 2,417 \\
\hline 18 & $75,00 \%$ & $100,00 \%$ & 4,667 & 7,000 & 2,250 & 4,083 \\
\hline 19 & $0,00 \%$ & $100,00 \%$ & 16,000 & 2,000 & 3,250 & 1,833 \\
\hline 20 & $75,00 \%$ & $83,33 \%$ & 8,667 & 4,000 & 2,750 & 2,083 \\
\hline Média & $48,75 \%$ & $76,25 \%$ & 9,400 & 3,100 & 2,200 & 1,933 \\
\hline Desvio Patrão & $26,78 \%$ & $29,26 \%$ & 6,270 & 1,921 & 0,917 & 0,865 \\
\hline
\end{tabular}

propagação de um determinado erro, evitando uma solução completa mas equivocada. A redução de erros é uma consequência de um benefício em relação ao processo de aquisição de conhecimento e pode-se confirmar esse aspecto como contribuição relevante da pesquisa apresentada.

\section{Considerações Finais}

O artigo presente propôs uma arquitetura para a elaboração de OAs que explora conceitos presentes nos STIs, como a remediação de erros e o rastreamento da interação do aluno com o ambiente, contando com o subsídio de MRE. Para demonstrar o uso da arquitetura proposta, foi criado, por meio da ferramenta de autoria FARMA, um OA que contemplasse os conceitos do Teorema de Pitágoras.

Com experimentos conduzidos sobre uso do Pitágoras Max, teve-se sucesso em validar o uso da remediação de erros para a aquisição dos conceitos matemáticos referidos. Tal remediação se sustenta na classificação de erros amparada em MRE.

Em adição, a arquitetura explorou o uso do modelo cognitivo baseado na Teoria ACT, que exige multiplicidade de passos. Isso foi provido pela possibilidade de estruturação do enunciado de um problema em etapas componentes do processo 


\section{CBIE-LACLO 2015}

Anais do XXVI Simpósio Brasileiro de Informática na Educação (SBIE 2015)

resolutivo. A ideia central foi que as etapas permitissem encontrar erros/equívocos do aluno, fornecendo a remediação como apoio para diminuí-los.

Os resultados apontam que há benefício, não apenas na aquisição de conhecimento, mas também na interação com os problemas do OA elaborado pela arquitetura proposta. Tais resultados provieram da comparação com a metodologia tradicional empregada para elaboração e resolução de problemas matemáticos.

\section{Referências}

Ainsworth, S. (2006). Deft: A conceptual framework for considering learning with multiple representations. Learning and Instruction, 16(3):183-198.

Anderson, J. R. (1983). The architecture of cognition. Psychology Press.

Bazzo, G. C., Marczal, D., and Direne, A. I. (2011). Classificação automática de erros de aprendizes humanos do processo de indução analítica. In Anais do Simpósio Brasileiro de Informática na Educação, pages 130-139.

Cox, R. and Brna, P. (1995). Supporting the use of external representations in problem solving: The need for flexible learning environments. Journal of Artificial Intelligence in Education, 6:239-302.

Leite, M. D., Marczal, D., and Pimentel, A. (2013). Multiple external representations in remediation of math errors. 15th International Conference on Enterprise Information Systems - ICEIS (2013), 1:540-544.

Leite, M. D., Marczal, D., Pimentel, A. R., and A., D. (2014). A conceptual framework to use remediation of errors based on multiple external remediation applied to learning objects. Journal of Technology and Science Education, North America, sep. 2014., 04(03).

Leite, M. D., Pimentel, A. R., and Pietruchinski, M. H. (2012). Remediação de erros baseada em múltiplas representações externas e classificação de erros aplicada a objetos de aprendizagem inteligentes. Anais do 23 Simpósio Brasileiro de Informática na Educação - SBIE. Rio de Janeiro.

Marczal, D. and Direne, A. I. (2011). Um arcabouço que enfatiza a retroação a contextos de erro na solução de problemas. Revista Brasileira de Informática na Educação, 19(01):63.

Nunes, T. M. and Jaques, P. A. (2014). Utilizando agentes pedagógicos animados como uma abordagem não restritiva ao gaming the system. Revista Brasileira de Informática na Educação, 22(01):147.

Oliveira, F. D., Leite, M. D., and Pimentel, A. R. (2012). Um processo de remediaçao de erros com base no uso de múltiplas representaçoes externas no contexto de objetos de aprendizagem. In Anais do Simpósio Brasileiro de Informática na Educação, volume 23.

Rau, M. A. and Scheines, R. (2012). Searching for variables and models to investigate mediators of learning from multiple representations. International Educational Data Mining Society. 